\title{
P02-08. Enhancement of HIV-I DNA vaccine immunogenicity by BCG-PSN, a novel adjuvant
} J Sun*, Y Liu, D Li, J Hou, Z Xu, W Fan, J Fu, Y Liu and Y Shao

Address: NCAIDS, China CDC, Beijing, PR China

* Corresponding author

from AIDS Vaccine 2009

Paris, France. 19-22 October 2009

Published: 22 October 2009

Retrovirology 2009, 6(Suppl 3):PI3 doi:10.1 I86/1742-4690-6-S3-PI3

This abstract is available from: http://www.retrovirology.com/content/6/S3/PI3

(C) 2009 Sun et al; licensee BioMed Central Ltd.

\section{Background}

Although the importance of DNA vaccines, especially as a priming immunization has been well established in numerous HIV vaccine studies, the immunogenicity of DNA vaccines is generally moderate. Novel adjuvant is in urgent need for improving the immunogenicity of DNA vaccine. Mycobacterium bovis bacillus Calmette-Guérin (BCG) cell-wall skeleton and nuclear acid which can activate at least five kinds of TLRs (TLR1, TLR2, TLR4, TLR6 and TLR9).

\section{Methods}

Polysaccharide nucleic acid fraction from BCG (BCGPSN) is an immuno-modulatory product extracted by hot phenol method from BCG which is considered to be successful clinical application in immune disorders or immune levels decline and the treatment of allergic diseases. In this study, we evaluated whether the BCG-PSN could serve as a novel adjuvant of DNA vaccine to elicit better cellular and humoral immune responses against the HIV-1 Env antigen in Balb/c mice model. The BCG-PSN was mixed with $10 \mu \mathrm{g}, 30 \mu \mathrm{g}, 100 \mu \mathrm{g}$ of pDRVI1.0gp1455M (HIV-1 CN54 gp145 gene) DNA vaccine and intramuscularly immunized two or three times.

\section{Results}

We found that BCG-PSN could significantly improve the immunogenicity of DNA vaccine when administered together with DNA vaccine. Further, we demonstrated that at $100 \mu \mathrm{g}$ dose, co-immunized twice with BCG-PSN adjuvant elicited Env specific immune responses which were comparable to those induced by three immuniza- tions with DNA alone. At the same vaccination schedule, BCG-PSN co-immunization with $30 \mu \mathrm{g}$ DNA vaccine could elicit cellular and humoral immune responses which were comparable to that induced by $100 \mu \mathrm{g}$ DNA vaccine alone.

\section{Conclusion}

BCG-PSN can serve as a novel adjuvant for DNA vaccination. 